\title{
A POINCARÉ-TYPE INEQUALITY ON THE EUCLIDEAN UNIT SPHERE
}

\author{
AI-JUN LI, YINGYING LOU AND YURONG JI
}

Abstract. We consider the second variation for the volume of convex bodies associated with the $L_{p}$ Minkowski-Firey combination and obtain a Poincaré-type inequality on the Euclidean unit sphere $S^{n-1}$.

Mathematics subject classification (2010): 52A20.

Keywords and phrases: Convex body, $L_{p}$-addition, Poincaré-type inequality.

\section{REFERENCES}

[1] A. D. Aleks androv, Konvexe Polyeder, Akademie-Verlag, Berlin (Russian original: 1950), 1958.

[2] S. Bов KOV, Isoperimetric and analytic inequalities for log-concave probability measures, Ann. Probability 27 (1999), 1903-1921.

[3] S. Bobkov, M. Ledoux, From Brunn-Minkowski to Brascamp-Lieb and to logarithmic Sobolev inequalities, Geom. Funct. Anal. 10 (2000), 1028-1052.

[4] S. Bob Kov, M. Ledoux, Weighted Poincaré-type inequalities for cauchy and other convex measures, Ann. Probability 37 (2009), 403-427.

[5] S. Bobkov, M. Ledoux, From Brunn-Minkowski to sharp Sobolev inequalities, Ann. Mat. Pure Appl. 187 (2008), 369-384.

[6] S. CAmpi, P. Gronchi, The $L_{p}$-Busemann-Petty centroid inequality, Adv. Math. 167 (2002), 128141.

[7] S. CAmpi, P. GRONCHI, On the reverse $L_{p}$-Busemann-Petty centroid inequality, Mathematika 49 (2002), 1-11.

[8] S.-Y. Cheng, S.-T. YAU, On the regularity of the solutions of the $n$-dimensional Minkowski problem, Comm. Pure Appl. Math. 29 (1976), 495-516.

[9] A. Coles ANTI, Brunn-Minkowski inequalities for variational problems and related problems, Adv. Math. 194 (2005), 105-140.

[10] A. Coles Anti, From the Brunn-Minkowski inequality to a class of Poincaré type inequalities, Commun. Contemp. Math. 10 (2008), 765-772.

[11] A. Coles Anti, E. Saorin-GomeZ, Functional inequalities derived from The Brunn-Minkowski inequality for quermassintegrals, J. Convex Anal. 17 (2010), 35-49,

[12] A. Coles Anti, I. Fragalà, The first variation of the total mass of log-concave functions and related inequalities, Adv. Math. 244 (2013), 708-749.

[13] W. J. FIREY, p-means of convex bodies, Math. Scand., 10 (1962), 17-24.

[14] R. J. Gardner, The Brunn-Minkowski inequality, Bull. Amer. Math. Soc. (N.S.) 39 (2002), 355-405.

[15] C. HABERL, $L_{p}$ intersection bodies, Adv. Math. 217 (2008), 2599-2624.

[16] C. Haberl, M. Ludwig, A characterization of $L_{p}$ intersection bodies, Int. Math. Res. Not. 17 (2006) Art. ID 10548, 29 pp.

[17] C. Haberl, F. Schuster, General $L_{p}$ affine isoperimetric inequalities, J. Differential Geom. 83 (2009), 1-26.

[18] C. Haberl, F. Schuster, Asymmetric affine $L_{p}$ Sobolev inequalities, J. Funct. Anal. 257 (2009), 641-658.

[19] M. Ludwig, Projection bodies and valuations, Adv. Math. 172 (2002), 158-168.

[20] M. Ludwig, Minkowski valuations, Trans. Amer. Math. Soc. 357 (2005), 4191-4213.

[21] M. Ludwig, Intersection bodies and valuations, Amer. J. Math. 128 (2006), 1409-1428. 
[22] E. LutwaK, The Brunn-Minkowski-Firey Theory I: Mixed volumes and the Minkowski Problem, J. Differential Geom. 38 (1993), 131-150.

[23] E. Lutwak, The Brunn-Minkowski-Firey Theory II, Adv. Math. 118 (1996), 244-294.

[24] E. Lutwak, D. YAng, G. Zhang, $L_{p}$ affine isoperimetric inequalities, J. Differential Geom. 56 (2000), 111-132,

[25] E. LutwaK, D. YAng, G. Zhang, Sharp affine $L_{p}$ Sobolev inequalities, J. Differential Geom. 62 (2002), 17-38.

[26] E. Lutwak, D. Yang, G. Zhang, On the $L_{p}$-Minkowski problem, Tran. Amer. Math. Soc. 356 (2004), 4359-4370.

[27] E. LutwaK, D. YAng, G. Zhang, Volume inequalities for subspaces of $L_{p}$, J. Differential Geom. 68 (2004), 159-184.

[28] E. Lutwak, D. Yang, G. Zhang, $L_{p}$ John ellipsoids, Proc. London Math. Soc. 90 (2005), $497-$ 520.

[29] M. Meyer, E. Werner, On the p-affine surface area, Adv. Math. 152 (2000), 288-313.

[30] R. SchneIder, Convex bodies: the Brunn-Minkowski theory, Encyclopedia of Mathematics and its Applications, Vol. 44, Cambridge University Press, Cambridge, 2014.

[31] E. Werner, D.-P. Ye, New $L_{p}$ affine isoperimetric inequalities, Adv. Math. 218 (2008), 762-780.

[32] G. ZHANG, The affine Sobolev inequality, J. Differential Geom. 53 (1999), 183-202. 